\title{
Effects of weight management by exercise modes on markers of subclinical atherosclerosis and cardiometabolic profile among women with abdominal obesity: a randomized controlled trial
}

\author{
Jina Choo ${ }^{1 *}$, Juneyoung Lee ${ }^{2}$, Jeong-Hyun Cho ${ }^{1}$, Lora E Burke ${ }^{3}$, Akira Sekikawa ${ }^{4}$ and Sae Young Jae ${ }^{5}$
}

\begin{abstract}
Background: Few studies have examined the differential effects of weight management by exercise mode on subclinical atherosclerosis. We hypothesized that 3 modes of aerobic, resistance, and combination exercises have differential effects on the flow-mediated dilation (FMD), carotid-femoral pulse wave velocity (PW), and carotid intima-media thickness (IMT) as well as cardiometabolic profile in weight management.

Methods: A randomized, single-blind trial (ISRCTN46069848) was conducted in Seoul, South Korea between November 2011 and December 2012. Randomized participants were 110 women with abdominal obesity (aerobic group $n=50$; resistance group $n=30$; combination exercise group $n=30$ ). The treatment period was 12 months with 3-month follow up: A diet-alone intervention for the first 3 months and a diet-plus-exercise intervention for the next 9 months according to exercise modes. The exercise training was designed with an intensity of 50-70\% heart rate reserve for 3 days a week in 60-minute-long sessions for 9 months, consisting of 30-minute treadmill and 30-minute bike exercises for aerobic group; upper and lower body exercises with an intensity target of 2 sets and 8-12 repetitions for resistance group; 30-minute resistance and consecutive 30-minute aerobic exercises for combination group.

Results: Ninety-two and 49 participants were analyzed for modified intention-to-treat analysis and per-protocol (PP) analysis, respectively. The 3 exercise modes had no significant differential effects on FMD, PWV, and IMT over time; however, the combination group was found to have significantly lower levels of fasting glucose than the aerobic group ( $p=.034)$ in the PP analysis. Nevertheless, we observed significant time effects such as reductions in PWV ( $p=.048)$ and IMT ( $p=.018)$ in cubic and quadratic trends, respectively, and improvements in body weight, waist circumference, low-density and high-density lipoprotein cholesterol levels, fasting glucose levels, and cardiorespiratory fitness in linear, quadratic, or cubic trends.

Conclusions: For women with abdominal obesity, a combination of aerobic and resistance exercises may be preferable to a single exercise mode for effective glucose control. Regardless of exercise mode, exercise interventions combined with dietary interventions in weight management may be beneficial in reducing the risk of subclinical atherosclerosis and cardiometabolic risk.
\end{abstract}

\section{Background}

Abdominal obesity is a risk factor for coronary heart disease (CHD). Prospective cohort studies have reported that an increase in waist circumference is significantly associated with CHD incidence and mortality [1]. In addition, abdominal obesity was found to be associated

* Correspondence: jinachoo@gmail.com

${ }^{1}$ College of Nursing, Korea University, Seoul, South Korea

Full list of author information is available at the end of the article with subclinical atherosclerotic risk, as assessed by endothelial dysfunction [2], aortic stiffness [3], and carotid atherosclerosis $[3,4]$. Particularly, the risk for CHD associated with increased waist circumference may be prominent in women [1,5-7], and, in this context, the potential for reducing CHD risk may also be significant in women.

Diet-plus-exercise interventions are commonly recommended for enhancing long-term weight management and reducing CHD risk factors in overweight and obese 
individuals $[8,9]$. Empirically, exercise-alone interventions have been found to have weaker effects than diet-plusexercise interventions on weight loss $[10,11]$. However, exercise training may have beneficial effects on the regression of subclinical atherosclerosis [12]. A few studies have reported that either aerobic or resistance exercise training improved endothelial dysfunction [13,14], aortic stiffness $[15,16]$, and carotid intima-media thickness (IMT) [17]. However, their effects may differ by exercise modes, i.e., aerobic, resistance, or combination exercise, because each mode leads to different patterns of blood flow and levels of pressure on the endothelium and arterial wall $[18,19]$. Meanwhile, recent studies have reported that a combination of aerobic and resistance exercises may be more effective on improving anthropometric and cardiometabolic profiles than aerobic or resistance exercise alone [20,21]. In particular, combination exercise was reported to be the most efficacious means of decreasing body weight and waist circumference among overweight and obese adults $[20,22]$ and to have an additional beneficial effect on glucose control compared with either aerobic or resistance exercise for those with type 2 diabetes mellitus [21]. However, there is no information regarding whether the 3 exercise modes, i.e., aerobic, resistance, and combination exercises, have differential effects on markers of subclinical atherosclerosis such as endothelial function, aortic stiffness, or carotid IMT as well as cardiometabolic profile among overweight and obese individuals.

The purpose of the study was to test the hypothesis that aerobic, resistance, and combination exercises in a weight management intervention would have significant differential effects on markers of subclinical atherosclerosis, as measured by brachial flow-mediated dilation (FMD), carotid-femoral pulse wave velocity (PWV) and mean IMT levels at the common carotid artery, and cardiometabolic profile among women with abdominal obesity in the Community-based Heart and Weight Management Trial.

\section{Methods}

\section{Study participants and enrollment procedure}

We recruited the study subjects from a community (i.e., Seongbuk-Gu) in Seoul, South Korea. The community was characterized as an urban county with a population size of approximately 490,000 residents. Between November 2010 and November 2011, study participants were recruited via poster, leaflet, telephone, and mass mailing advertisements at the municipal health center, churches, universities, and online communities. Eligible participants were invited to an orientation where they were provided a detailed study overview, asked for consent, and screened for additional inclusion criteria. The inclusion criteria were as follows: healthy women aged between 18 and 65 years, elevated waist circumference $(\geq 85 \mathrm{~cm})$ according to the criteria for abdominal obesity as defined by the Korean Society for the Study of Obesity $[23,24]$, and willingness to be randomly assigned to one of the 3 different exercise modes (i.e., aerobic, resistance, and combination exercise training). The exclusion criteria included current medical conditions such as cardiovascular diseases, diabetes, or cancers; physical limitations restricting exercise ability; current use of hormone therapy; history of participation in a weight loss intervention in the last 1 year; and a weight change in the last 4 weeks prior to participation in our study. Before commencing with the randomization of participants, baseline measurements of body composition (body mass index [BMI] and waist circumference), blood lipids, and fasting glucose were obtained; information regarding sociodemographic and psychosocial variables were obtained through questionnaires. The study was approved by the Institutional Review Board at Korea University (KU-IRB-11-10-A-2). All participants provided written informed consent.

\section{Study design}

The Community-based Heart and Weight Management Trial (trial registration no. ISRCTN46069848) includes a randomized 12-month intervention with 3 different exercise groups: aerobic training only, resistance training only, and a combination of aerobic and resistance training. The intervention assignment was carried out using a random allocation computer program ( $\mathrm{n}=50$ for aerobic exercise, $\mathrm{n}=30$ for resistance exercise, and $\mathrm{n}=30$ for combination exercise) (Figure 1). Initially, the aerobic exercise group $(\mathrm{n}=50)$ was further divided into 2 groups: one group with $(n=30)$ and the other group without behavioral therapy $(n=20)$. However, because there were no significant differences in the outcome variables between both the groups, we pooled the participants of those 2 groups into 1 aerobic exercise group $(n=50)$ for the present study. This study was a single-blinded trial; assessors of all the outcomes were blinded to participant group assignment, and all outcome data were blinded until the completion of final data entry for the 12-month assessment.

\section{Intervention}

We conducted 2 consecutive types of interventions: dietalone vs. diet-plus-exercise interventions in a sequence over a 12-month weight management program (Figure 2), i.e., a diet-alone intervention for the first 3 months and a diet-plus-exercise intervention for the next 9 months according to exercise modes; for the diet-alone intervention, the 3 groups were asked not to attend any exercise training sessions for the first 3 months.

The exercise intervention over the following 9 months was added to the diet-alone intervention: 60-minute exercise sessions were conducted 3 times a week in a public 


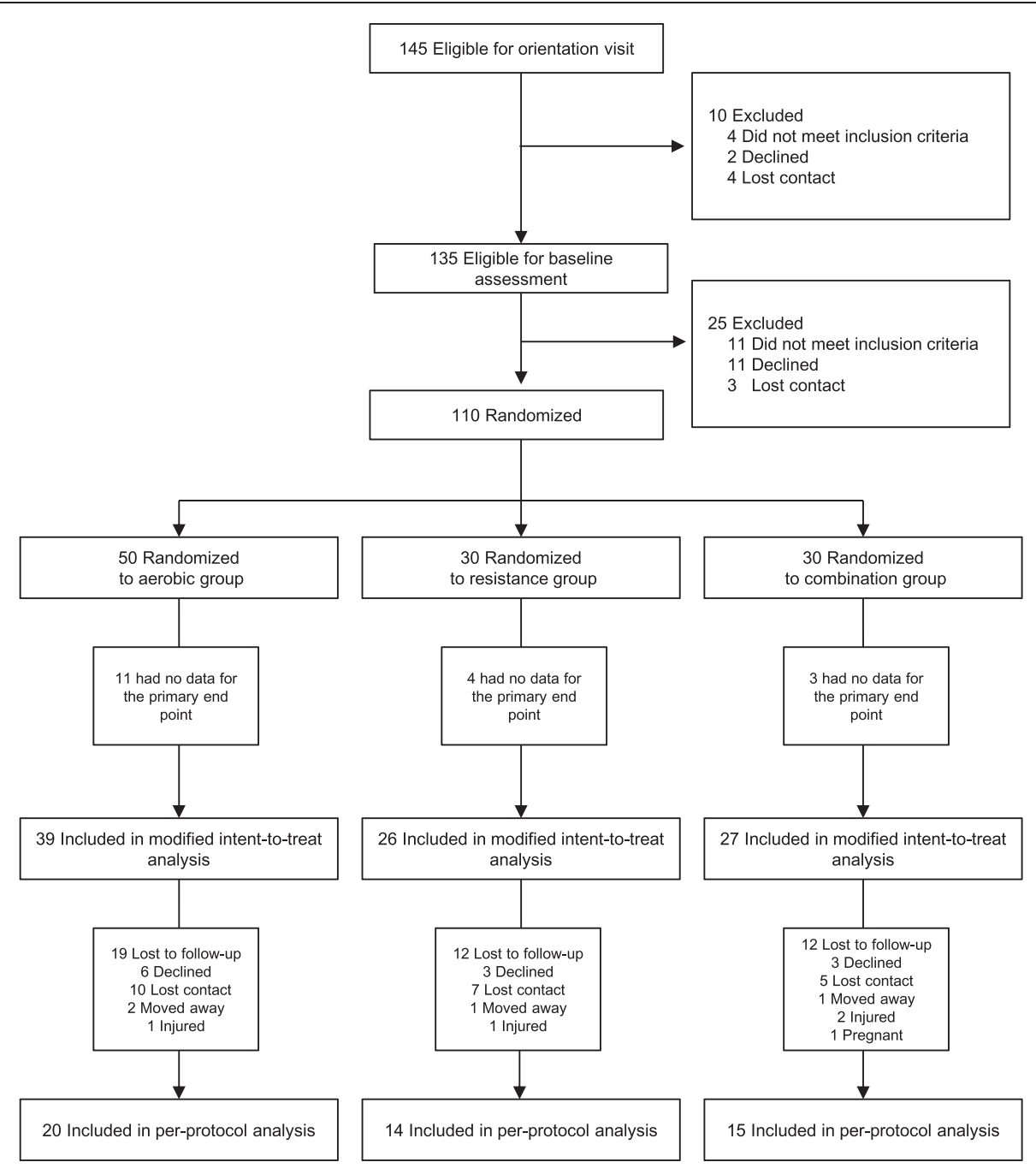

Figure 1 Participant flow chart.

community fitness center under group teaching and supervision of exercise trainers. The aerobic session consisted of 30-minute treadmill exercise and 30-minute bike exercise, with an intensity target of $50-70 \%$ of heart rate reserve. The resistance session consisted of both upper and lower body exercises: chest press, lat pulldown, abdominal crunches, back extensions, leg curl, leg extensions, leg press, and calf raise. The load was initially set at $40 \%$ and $50 \%$ (or $60 \%$ ) of maximum strength for the upper extremities and lower extremities, respectively. Two sets of 8-12 repetitions were performed during each session. The rest period between each set was less than 90 seconds. The load was progressively increased by $5 \%$ every 3 weeks or when subjects could easily perform 15 repetitions. Combination training consisted of 30 minutes of resistance exercise and a consecutive 30-minute session of aerobic exercise (15 minutes on a treadmill and 15 minutes on a bicycle) with 1 set of $8-12$ repetitions for resistance exercise and $50-70 \%$ of the heart rate reserve for aerobic exercise.

The diet-alone intervention was administered to all participants, with individualized daily calorie intake $(1,200 \mathrm{kcal}$ if the weight was $<90.5 \mathrm{~kg} ; 1,500 \mathrm{kcal}$ if the weight was $\geq 90.5 \mathrm{~kg})$ and fat intake goals $(\leq 25 \%$ of total calories), taking into account their baseline body weight measurements. Additionally, all the groups except the aerobic group without behavioral therapy $(n=20)$ received behavioral counseling (12 sessions of group classes over 6 months) and were also taught the use of established behavior change strategies including goal setting and self-monitoring [25].

\section{Measurements}

The primary outcome of the trial was FMD. The secondary outcomes included improvements in other markers of subclinical atherosclerosis (i.e., PWV and carotid IMT), 
anthropometric profile (i.e., body weight and lean body mass), cardiometabolic profile (i.e., waist circumference, systolic blood pressure, total cholesterol, low-density lipoprotein [LDL] cholesterol, high-density lipoprotein [HDL] cholesterol, triglycerides, and fasting glucose), and cardiorespiratory fitness (i.e., maximal oxygen consumption $\left.\left[\mathrm{VO}_{2 \mathrm{max}}\right]\right)$.

All measures were assessed at baseline, 3, 6, and 12 months. Weight was measured after an overnight fast, using the Tanita bioelectrical impedance scale (Tanita Corporation of America, Inc., IL, USA); prior to being weighed, the participants were asked to wear lightweight clothing and take off their shoes. Height was measured using a wall-mounted stadiometer. The BMI was computed as weight $(\mathrm{kg}) /$ height $(\mathrm{m})^{2}$. Waist circumference (cm) was measured twice using a Gullick II measuring tape at the midpoint between the lowest rib and the iliac crest; the average of 2 measurements was used.

Blood samples were drawn from the antecubital vein in the morning after a 10-hour overnight fast without taking any current medications, including anti-hypertensive or lipid-lowering medications, when participants visited the municipal health center. Participants who took antihypertensive $(n=11)$ or lipid-lowering medications $(n=7)$ were instructed to take them during the study period. Samples were analyzed using a biochemical auto-analyzer (Hitachi cobas c501; Hitachi, Japan) at the Department of Clinical Laboratory of the municipal health center, in Seoul, South Korea. Total cholesterol and HDL cholesterol levels were measured by a homogeneous enzymatic colorimetric test. The levels of triglycerides and glucose were measured using the GPO-PAP method and the hexokinase method, respectively. LDL cholesterol level was estimated according to the Friedewald equation [26].

For non-invasive assessment of the FMD, brachial artery dilatation was measured by ultrasonography (ACUSON X 300; Siemens, Mountain View, CA). The brachial artery was imaged in longitudinal sections $5-10 \mathrm{~cm}$ proximal to the placement of a blood pressure cuff, just below the antecubital fossa, using a high frequency linear array probe (11.4 MHz). The FMD was measured for 1 minute at baseline and again for 2 minutes after an ischemic stimulus (inflation of a blood pressure cuff around the forearm to $200 \mathrm{mmHg}$ for 5 minutes). The FMD was analyzed using a semi-automated edge detection software system (Brachial Analyzer 5; Medical Imaging Applications, Coralville, IA) to calculate FMD (\%) according to the following equation: [(maximum diameter - baseline diameter)/baseline diameter $\times 100$. A reproducibility (i.e., intraclass correlation coefficient) of 0.95 was achieved within technicians.

Carotid to femoral PWV value was derived to measure central arterial stiffness, using a SphygmoCor ${ }^{\oplus}$ system (AtCor Medical, Sydney, Australia). The distance from the carotid artery sample site to the femoral artery and that from the carotid artery to the suprasternal notch were measured as straight lines with a tape measure. Pulse waves were obtained by applanation tonometry. According to the "foot-to-foot" method, PWV was determined as $\mathrm{D} /(\Delta \mathrm{t})(\mathrm{m} / \mathrm{s})$, where $\Delta \mathrm{t}$ is the time difference between carotid-femoral pressure waves, and $\mathrm{D}$ is the distance between the 2 arteries. All measurements 
were taken in duplicate, and the mean values were used for subsequent analysis. Arterial stiffness was measured in accordance with the guidelines of the Clinical Application of Arterial Stiffness, Task Force III. A reproducibility (i.e., intraclass correlation coefficient) of 0.98 was achieved within technicians.

Carotid artery ultrasound imaging was performed using a high-resolution B-mode ultrasound system (ACUSON X 300; Siemens, Mountain View, CA). The IMT was defined as the distance from the leading edge of the lumen-intima interface to the leading edge of the media-adventitia interface of the far wall of the carotid artery. All measurements were made at the end diastole. The carotid IMT of the common carotid artery was determined from a semiautomated measurement obtained $2 \mathrm{~cm}$ proximal to the carotid bifurcation. The value for carotid IMT was defined as the mean of the IMT. A reproducibility (i.e., intraclass correlation coefficient) of 0.99 was achieved within technicians.

Cardiorespiratory fitness levels were reported as $\mathrm{VO}_{2 \max }$ values estimated using the Rockport Fitness Walking Test. Participants were administered the 1-mile walk test on an outdoor track in accordance with the protocol of Kline et al. [27].

\section{Statistical analysis}

Sample size calculation was based on Olson et al.'s study [13]. The study showed a decreased mean FMD change of $1.4 \%$ (standard deviation [SD], 2.32\%) at the 12-month follow-up in the control group and an increased mean FMD change of $2.6 \%$ (SD, 3.49\%) in the resistance group. We considered two-thirds of mean change difference between groups as an effect size for our study because all of our groups received a diet program. To detect a mean difference in FMD of $2.67 \%$ by assuming the SD from Olson et al., between any pairs of the 3 groups in our study, at least 21 subjects per group were needed to ensure an $80 \%$ power with the Student's $t$-test and 2-tailed test at the 5\% level of significance. Considering the 30\% follow-up loss rate during the study period, we initially planned to randomize a total of 90 subjects to 3 study groups (30 participants per group). However, as mentioned in the Study Design section, the actually allocated sample sizes were 50 participants to aerobic, 30 participants to resistance, and 30 participants to the combination exercise group.

To test the homogeneity of exercise groups in terms of baseline measures for sociodemographic, health-related, subclinical atherosclerosis, anthropometric, cardiometabolic, and cardiorespiratory fitness variables, one-way ANOVA, $\mathrm{X}^{2}$ test, and Fisher's exact test were performed. The modified intention-to-treat (ITT) population, defined as randomized subjects with least 1 FMD measure reported across 4 assessment periods, was used to analyze the primary outcome variable. This population consisted of $92(84 \%)$ out of 110 randomized participants (Figure 1). The main effects of exercise group and time as well as the interaction effects between exercise group and time were examined using a generalized estimating equation (GEE) model for repeated measures over time. Covariates adjusted for in the GEE model included baseline values and marital status. Per-protocol (PP) analyses were also conducted; the participants involved in this analysis were defined as a subgroup of participants who met the criteria of $100 \%$ follow-up by the fifth assessment over 12 months.

All analyses were performed using STATA 10.0 (StataCorp LP., USA). A 2-tailed p-value of less than 0.05 was considered statistically significant.

\section{Results}

The participants had a mean age of 43.1 years, mean BMI of $28.5 \mathrm{~kg} / \mathrm{m}^{2}$, and mean waist circumference of $94.8 \mathrm{~cm}$ (Table 1). Of all participants, $24.5 \%$ had an obesity-related condition such as hypertension or dyslipidemia and $29.1 \%$ were post-menopausal. Some of the baseline cardiometabolic profile (i.e., waist circumference and total and HDL cholesterol levels) were outside the reference ranges according to the criteria for abdominal obesity in women as defined by the Korean Society for the Study of Obesity [23], criteria of the National Cholesterol Education Program (NCEP) Adult Treatment Panel III (ATP III) [28], and diagnostic criteria for metabolic syndrome according to the NCEP-ATP III [29]. The mean fitness level, as measured by $\mathrm{VO}_{2 \max }$, was $31.1 \mathrm{~mL} \cdot \mathrm{kg}^{-1}$. $\mathrm{min}^{-1}$. The participants in the resistance group were highly likely to be married compared to those in the aerobic or combination exercise groups $(\mathrm{F}=6.57, \mathrm{p}=.037)$.

Table 2 summarizes the results for differential effects of exercise mode on outcome variables over time in weight management, i.e., the interaction effects between exercise groups and time on markers of subclinical atherosclerosis and anthropometric and cardiometabolic profiles on the basis of both modified ITT and PP analyses. We did not find significant interaction effects for any of the outcome variables, except for fasting glucose levels. The PP analyses revealed that changes in fasting glucose levels significantly differed by the exercise mode $(\mathrm{p}=.034)$; significant reductions in fasting glucose levels were seen in the combination exercise group as compared with that in the aerobic group. Specifically, an additional analysis revealed that such a significant differential effect occurred at 12 months $(\mathrm{p}=.004)$, and the changes in fasting glucose levels in the combination exercise group showed a liner trend $(\mathrm{p}=.014)$ (data not shown).

Table 3 summarizes the time effects for all the 3 groups on the outcome variables, based on the results of modified ITT and PP analyses. We found significant time effects for PWV $(\mathrm{p}=.048)$ and IMT $(\mathrm{p}=.018)$. We also 
Table 1 Participants' characteristics $(\mathrm{N}=110)$

\begin{tabular}{|c|c|c|c|c|c|c|}
\hline & \multicolumn{4}{|c|}{$\mathrm{n}(\%)$ or mean (SD) } & \multirow{2}{*}{$\begin{array}{c}\mathrm{F} / \mathrm{X}^{2} / \\
\text { Fisher's }\end{array}$} & \multirow[b]{2}{*}{$P^{*}$} \\
\hline & $\begin{array}{l}\text { Total } \\
(n=110)\end{array}$ & $\begin{array}{l}\text { Aerobic } \\
(n=50)\end{array}$ & $\begin{array}{l}\text { Resistance } \\
(n=30)\end{array}$ & $\begin{array}{l}\text { Combination } \\
(\mathbf{n}=30)\end{array}$ & & \\
\hline \multicolumn{7}{|l|}{ Sociodemographic characteristics } \\
\hline Age, years & $43.1(9.0)$ & $42.2(9.5)$ & $46.0(10.0)$ & $41.8(6.6)$ & 2.16 & .120 \\
\hline Married, yes & $90(81.8)$ & $37(74.0)$ & $29(97.6)$ & $24(80.0)$ & 6.57 & .037 \\
\hline Education & & & & & 0.78 & .678 \\
\hline Some college or more & $66(60.0)$ & $31(62.0)$ & $16(53.3)$ & $19(63.3)$ & & \\
\hline High school degree or less & $44(40.0)$ & $19(38.0)$ & $14(46.7)$ & $11(36.7)$ & & \\
\hline Monthly household income (\$) & & & & & 1.37 & .503 \\
\hline$<=3,500$ & $61(55.5)$ & $30(60.0)$ & $14(46.7)$ & $17(56.7)$ & & \\
\hline$>3,500$ & $49(44.5)$ & $20(40.0)$ & $16(53.3)$ & $13(43.3)$ & & \\
\hline Employed, yes & $43(39.1)$ & $19(38.0)$ & $11(36.7)$ & $13(43.3)$ & 0.33 & .850 \\
\hline \multicolumn{7}{|l|}{ Health-related characteristics } \\
\hline Obesity-related conditions, yes & $27(24.5)$ & $15(30.0)$ & $8(26.7)$ & $4(13.3)$ & 2.91 & .233 \\
\hline Post-menopause, yes & $32(29.1)$ & $15(30.0)$ & $12(40.0)$ & $5(16.7)$ & 4.00 & .136 \\
\hline Current smoking, yes & $6(5.5)$ & $2(4.0)$ & $1(3.3)$ & $3(10.0)$ & 1.57 & .556 \\
\hline Alcohol drinking (twice or more/week), yes & $9(8.2)$ & $4(8.0)$ & $2(6.7)$ & $3(10.0)$ & 0.36 & 1.000 \\
\hline Anti-hypertensive medication, yes & $11(10.0)$ & $7(14.0)$ & $4(13.3)$ & $0(0.0)$ & 5.22 & .101 \\
\hline Lipid-lowering medication, yes & $7(6.4)$ & $0(0.0)$ & $5(16.7)$ & $2(6.7)$ & 8.74 & .007 \\
\hline \multicolumn{7}{|l|}{ Markers of subclinical atherosclerosis } \\
\hline FMD, \% & $10.76(3.457)$ & $11.10(3.263)$ & $9.94(3.477)$ & $11.08(3.723)$ & 1.18 & .310 \\
\hline $\mathrm{PW}, \mathrm{m} / \mathrm{sec}$ & $7.86(1.356)$ & $7.77(1.615)$ & $7.89(1.210)$ & $7.99(1.005)$ & 0.24 & .784 \\
\hline IMT, mm & $0.67(0.128)$ & $0.68(0.118)$ & $0.68(0.131)$ & $0.66(0.142)$ & 0.32 & .724 \\
\hline \multicolumn{7}{|l|}{ Anthropometric profile } \\
\hline Body weight, kg & $72.4(10.55)$ & $72.3(9.94)$ & $70.8(10.25)$ & $74.2(11.86)$ & 0.77 & .467 \\
\hline Body mass index, $\mathrm{kg} / \mathrm{m}^{2}$ & $28.5(0.36)$ & $28.5(0.54)$ & $27.9(0.58)$ & $29.1(0.79)$ & 0.79 & .456 \\
\hline Lean body mass, kg & $46.0(4.76)$ & $46.0(4.69)$ & $45.5(5.11)$ & $46.4(4.63)$ & 0.25 & .776 \\
\hline \multicolumn{7}{|l|}{ Cardiometabolic profile } \\
\hline Waist circumference, $\mathrm{cm}$ & $94.8(7.80)$ & $94.4(7.00)$ & $93.8(7.25)$ & $96.4(9.46)$ & 0.93 & .397 \\
\hline Systolic BP, mmHg & $116.5(13.08)$ & $116.6(12.97)$ & $117.4(10.38)$ & $115.6(15.78)$ & 0.15 & .859 \\
\hline Total cholesterol, mg/dL & $212.1(39.74)$ & $214.8(37.51)$ & $204.5(45.99)$ & $215.2(36.81)$ & 0.75 & .476 \\
\hline LDL-cholesterol, mg/dL & $129.1(36.39)$ & $130.3(32.46)$ & $120.2(42.98)$ & $135.9(34.75)$ & 1.47 & .235 \\
\hline HDL-cholesterol, mg/dL & $53.1(12.61)$ & $53.0(12.59)$ & $51.6(15.14)$ & $54.6(9.78)$ & 0.44 & .642 \\
\hline Triglycerides, mg/dL & $131.2(98.40,73.50)$ & $147.1(97.10,205.65)$ & $136.5(105.05,177.15)$ & $122.3(96.13,146.93)$ & 2.16 & .120 \\
\hline Fasting glucose, mg/dL & $89.6(14.45)$ & $90.1(16.72)$ & $93.6(14.50)$ & $84.9(8.05)$ & 2.89 & .060 \\
\hline \multicolumn{7}{|l|}{ Dietary intake $^{a}$} \\
\hline Total energy (kcal/day) & $1538.2(446.01)$ & 1498.9 (441.99) & $1552.3(360.01)$ & $1585.8(525.19)$ & 0.33 & .717 \\
\hline Carbohydrates (g/day) & $218.5(64.91)$ & $217.5(74.77)$ & $216.8(40.41)$ & $221.6(68.26)$ & 0.05 & .956 \\
\hline Protein (g/day) & $65.4(25.62)$ & $61.9(18.10)$ & $66.6(23.40)$ & $69.6(35.68)$ & 0.82 & .445 \\
\hline Lipid (g/day) & $45.6(18.23)$ & $43.3(17.53)$ & $46.4(15.78)$ & $48.4(21.27)$ & 0.68 & .508 \\
\hline \multicolumn{7}{|l|}{ Cardiorespiratory fitness } \\
\hline $\mathrm{VO}_{2 \max }, \mathrm{mL} / \mathrm{kg} / \mathrm{min}$ & $31.1(6.24)$ & $31.3(5.80)$ & $30.6(6.16)$ & $31.3(7.15)$ & 0.16 & .849 \\
\hline
\end{tabular}

$\mathrm{BP}=$ blood pressure; FMD = flow mediated dilation; $\mathrm{HDL}$ = high-density lipoprotein; $\mathrm{LDL}=$ low-density lipoprotein; $\mathrm{PWV}=$ pulse wave velocity; IMT = intima media thickness; $\mathrm{SD}=$ standard deviation; $\mathrm{VO}_{2 \max }=$ maximal oxygen consumption; Obesity-related conditions include hypertension and dyslipidemia.

${ }^{*} \mathrm{P}$-values from one-way ANOVA, chi-square or Fisher's exact test as appropriate a Analyzed with the sample size $(\mathrm{N}=96)$. 
Table 2 Differential effects of exercise mode on markers of subclinical atherosclerosis and anthropometric and cardiometabolic profiles over time

\begin{tabular}{|c|c|c|c|c|c|c|c|c|}
\hline & Modified & tention-to-trea & analysis $(\mathrm{N}=92)$ & & & r-protocol anal & $s(N=49)$ & \\
\hline & & Mean (SD) & & & & Mean (SD) & & \\
\hline & $\begin{array}{l}\text { Aerobic } \\
(n=39)\end{array}$ & $\begin{array}{l}\text { Resistance } \\
(n=26)\end{array}$ & $\begin{array}{l}\text { Combination } \\
(n=27)\end{array}$ & $P^{a}$ & $\begin{array}{l}\text { Aerobic } \\
(n=20)\end{array}$ & $\begin{array}{l}\text { Resistance } \\
(n=14)\end{array}$ & $\begin{array}{l}\text { Combination } \\
(n=15)\end{array}$ & $P^{a}$ \\
\hline FMD (\%) & & & & .792 & & & & .847 \\
\hline $3 \mathrm{M}$ & 11.28 (3.50) & $10.32(3.80)$ & $11.02(3.49)$ & & $11.51(4.02)$ & $10.75(3.67)$ & 11.32 (3.37) & \\
\hline $6 \mathrm{M}$ & $11.55(3.80)$ & $11.22(4.43)$ & $11.10(3.40)$ & & $12.38(4.43)$ & 11.02 (4.39) & 11.46 (3.74) & \\
\hline $9 \mathrm{M}$ & $11.08(4.05)$ & 10.89 (4.33) & $12.41(4.27)$ & & $11.34(4.88)$ & $10.05(4.11)$ & 13.18 (4.34) & \\
\hline $12 \mathrm{M}$ & $10.70(3.75)$ & 11.54 (4.99) & $11.30(4.04)$ & & $10.56(4.15)$ & 11.26 (5.49) & 11.20 (4.09) & \\
\hline PWV (m/sec) & & & & .585 & & & & .732 \\
\hline $3 \mathrm{M}$ & 7.91 (1.18) & $7.62(1.16)$ & $8.11(1.05)$ & & $7.51(0.77)$ & $7.49(0.82)$ & $7.81(1.04)$ & \\
\hline $6 \mathrm{M}$ & $7.83(1.31)$ & $7.52(1.15)$ & $7.76(1.04)$ & & $7.36(0.94)$ & $7.34(0.75)$ & 7.37 (0.98) & \\
\hline $9 M$ & $7.94(1.32)$ & 7.70 (1.18) & $7.96(1.01)$ & & 7.53 (1.15) & $7.60(0.83)$ & $7.59(1.02)$ & \\
\hline $12 \mathrm{M}$ & 7.88 (1.30) & 7.74 (1.17) & $7.96(0.89)$ & & $7.45(1.00)$ & $7.67(0.81)$ & $7.61(0.80)$ & \\
\hline IMT (mm) & & & & .574 & & & & .379 \\
\hline $3 \mathrm{M}$ & $0.71(0.19)$ & $0.72(0.17)$ & $0.66(0.10)$ & & $0.74(0.24)$ & $0.72(0.19)$ & $0.65(0.09)$ & \\
\hline $6 \mathrm{M}$ & $0.68(0.15)$ & $0.67(0.14)$ & $0.64(0.12)$ & & $0.71(0.18)$ & $0.65(0.14)$ & $0.65(0.13)$ & \\
\hline $9 M$ & $0.69(0.14)$ & $0.69(0.14)$ & $0.67(0.10)$ & & $0.73(0.16)$ & $0.69(0.13)$ & $0.67(0.10)$ & \\
\hline $12 \mathrm{M}$ & $0.70(0.14)$ & $0.70(0.13)$ & $0.67(0.11)$ & & $0.73(0.15)$ & $0.71(0.11)$ & $0.68(0.12)$ & \\
\hline Body weight (kg) & & & & .539 & & & & .894 \\
\hline $3 \mathrm{M}$ & 71.11 (10.69) & $67.20(9.36)$ & $72.62(12.06)$ & & $69.48(11.63)$ & 64.19 (5.59) & 69.06 (8.69) & \\
\hline $6 \mathrm{M}$ & $69.48(11.70)$ & $65.70(9.45)$ & $71.30(12.25)$ & & $67.14(12.84)$ & $62.71(6.27)$ & $66.93(7.74)$ & \\
\hline $9 M$ & $69.31(11.81)$ & $65.60(9.29)$ & $71.69(12.25)$ & & 65.25 (8.75) & $62.64(6.26)$ & $67.15(7.87)$ & \\
\hline $12 \mathrm{M}$ & $69.34(11.84)$ & 65.79 (9.34) & $71.75(12.63)$ & & $67.23(12.70)$ & $62.99(6.57)$ & 67.25 (8.98) & \\
\hline Lean body mass (kg) & & & & .717 & & & & .637 \\
\hline $3 \mathrm{M}$ & $45.41(4.96)$ & $44.44(5.09)$ & $45.94(4.95)$ & & $44.49(5.88)$ & $43.50(4.92)$ & $44.60(3.81)$ & \\
\hline $6 \mathrm{M}$ & $45.06(5.28)$ & $44.46(4.90)$ & $45.90(4.83)$ & & $44.95(6.34)$ & $43.62(4.69)$ & 44.38 (3.53) & \\
\hline $9 \mathrm{M}$ & $45.03(5.18)$ & $44.30(5.02)$ & $45.90(4.62)$ & & $44.25(4.62)$ & $43.62(5.05)$ & $44.36(3.24)$ & \\
\hline $12 \mathrm{M}$ & $44.94(5.14)$ & 44.29 (4.73) & $45.74(4.76)$ & & $44.91(6.04)$ & $43.61(4.48)$ & $44.07(3.45)$ & \\
\hline WC $(\mathrm{cm})$ & & & & .790 & & & & .825 \\
\hline $3 \mathrm{M}$ & $93.44(8.06)$ & $90.14(8.04)$ & $94.42(11.36)$ & & $91.32(8.00)$ & $87.26(4.72)$ & $90.41(7.11)$ & \\
\hline $6 \mathrm{M}$ & $91.39(9.41)$ & $87.87(8.56)$ & $92.83(12.00)$ & & 88.59 (9.67) & $84.54(5.25)$ & 87.46 (7.38) & \\
\hline $9 \mathrm{M}$ & $90.77(9.82)$ & $88.12(8.11)$ & $93.61(11.68)$ & & 87.61 (8.99) & $85.21(4.68)$ & 88.39 (6.99) & \\
\hline $12 \mathrm{M}$ & 91.27 (9.33) & $88.78(7.98)$ & 94.09 (11.58) & & $89.18(9.24)$ & $86.44(4.97)$ & $89.28(7.33)$ & \\
\hline Systolic BP (mmHg) & & & & .935 & & & & .838 \\
\hline $3 \mathrm{M}$ & 115.36 (15.84) & 113.10 (11.99) & 113.94 (17.98) & & $114.18(12.81)$ & $109.07(9.46)$ & 110.43 (13.70) & \\
\hline $6 \mathrm{M}$ & $113.06(15.46)$ & $112.37(10.23)$ & $112.74(17.79)$ & & $111.03(14.66)$ & 111.29 (12.10) & 107.53 (11.93) & \\
\hline $9 \mathrm{M}$ & $114.62(14.55)$ & $113.21(9.53)$ & $112.48(17.42)$ & & $113.33(13.19)$ & $110.79(8.74)$ & $106.60(8.01)$ & \\
\hline $12 \mathrm{M}$ & $113.95(14.41)$ & 111.94 (10.29) & 112.91 (18.64) & & $112.03(12.17)$ & $108.43(9.49)$ & $107.37(12.45)$ & \\
\hline TC (mg/dL) & & & & .517 & & & & .689 \\
\hline $3 \mathrm{M}$ & $197.111(44.28)$ & 192.13 (32.70) & 201.30 (34.03) & & 205.07 (51.68) & $185.94(28.42)$ & 191.34 (38.60) & \\
\hline $6 \mathrm{M}$ & $195.14(36.26)$ & 192.95 (35.23) & 202.67 (38.54) & & 198.05 (39.55) & 186.59 (28.53) & 190.07 (42.89) & \\
\hline $9 \mathrm{M}$ & 201.65 (37.10) & 202.18 (40.98) & 207.30 (38.77) & & $205.51(40.42)$ & 203.40 (41.73) & 195.81 (43.64) & \\
\hline $12 \mathrm{M}$ & $198.86(42.22)$ & $197.64(41.46)$ & $201.83(40.73)$ & & $202.53(50.23)$ & $193.34(42.04)$ & 185.39 (43.74) & \\
\hline
\end{tabular}


Table 2 Differential effects of exercise mode on markers of subclinical atherosclerosis and anthropometric and cardiometabolic profiles over time (Continued)

\begin{tabular}{|c|c|c|c|c|c|c|c|c|}
\hline LDL-C (mg/dL) & & & & .458 & & & & .300 \\
\hline $3 \mathrm{M}$ & 119.22 (38.47) & $114.40(29.22)$ & 121.88 (36.97) & & $128.36(47.15)$ & $111.60(31.37)$ & 110.59 (40.99) & \\
\hline $6 \mathrm{M}$ & 116.65 (30.49) & $115.46(28.85)$ & 124.18 (35.65) & & $121.73(35.34)$ & $110.64(27.77)$ & 112.96 (38.75) & \\
\hline $9 \mathrm{M}$ & 121.99 (32.78) & 118.19 (39.83) & 129.31 (39.32) & & 127.99 (37.16) & $117.66(46.93)$ & 117.65 (42.97) & \\
\hline $12 \mathrm{M}$ & 116.85 (36.74) & 116.70 (39.86) & 123.48 (39.58) & & $123.66(44.04)$ & 113.70 (46.65) & 106.80 (39.76) & \\
\hline $\mathrm{HDL}-\mathrm{C}(\mathrm{mg} / \mathrm{dL})$ & & & & .973 & & & & .562 \\
\hline $3 \mathrm{M}$ & $52.91(12.29)$ & $49.25(12.57)$ & $53.58(10.09)$ & & $52.27(12.55)$ & $46.98(13.77)$ & $54.99(11.10)$ & \\
\hline $6 \mathrm{M}$ & $53.74(13.04)$ & 53.60 q(13.93) & $54.79(10.69)$ & & $53.70(14.45)$ & $51.99(16.37)$ & $56.19(12.54)$ & \\
\hline $9 \mathrm{M}$ & $54.52(12.48)$ & $55.68(14.20)$ & $56.20(10.07)$ & & $55.78(13.15)$ & $56.04(16.64)$ & $59.19(11.69)$ & \\
\hline $12 \mathrm{M}$ & $57.72(15.15)$ & $54.69(13.17)$ & $56.39(10.35)$ & & $59.09(14.98)$ & $53.51(14.94)$ & $59.39(11.96)$ & \\
\hline Triglycerides (mg/dL) & & & & & & & & .784 \\
\hline $3 \mathrm{M}$ & $124.90(59.18)$ & $142.40(75.42)$ & 129.23 (100.99) & & $122.18(43.31)$ & 136.77 (96.43) & $128.83(128.67)$ & \\
\hline $6 \mathrm{M}$ & $123.74(71.06)$ & 119.47 (57.73) & $118.53(56.25)$ & & 113.06 (57.94) & $119.85(69.02)$ & $104.58(58.74)$ & \\
\hline $9 \mathrm{M}$ & 125.72 (68.76) & 141.51 (91.41) & 108.93 (50.78) & & 108.70 (44.08) & $148.51(120.31)$ & $94.87(52.08)$ & \\
\hline $12 \mathrm{M}$ & $120.88(70.84)$ & 131.24 (82.54) & 109.78 (48.56) & & $97.81(38.99)$ & 130.63 (107.88) & $96.03(48.36)$ & \\
\hline Fasting glucose (mg/dL) & & & & .413 & & & & .034 \\
\hline $3 \mathrm{M}$ & $88.93(9.87)$ & $94.54(19.83)$ & 89.39 (7.69) & & $86.40(7.51)$ & $93.54(12.40)$ & $88.52(7.78)$ & \\
\hline $6 \mathrm{M}$ & $85.80(7.90)$ & $91.68(19.82)$ & $85.67(7.47)$ & & $83.85(6.56)$ & $89.711(12.61)$ & $82.56(7.86)$ & \\
\hline $9 \mathrm{M}$ & $84.98(9.76)$ & $93.16(22.15)$ & $84.97(8.23)$ & & $82.33(9.23)$ & $94.61(17.40)$ & $81.33(8.53)$ & \\
\hline $12 \mathrm{M}$ & $89.78(8.28)$ & $92.84(22.41)$ & $86.30(7.22)$ & & $89.26(7.07)$ & $93.54(18.33)$ & $81.91(5.95)^{*}$ & \\
\hline \multicolumn{9}{|l|}{$\mathrm{VO}_{2 \max }(\mathrm{mL} / \mathrm{kg} / \mathrm{min})$} \\
\hline $3 \mathrm{M}$ & $31.77(5.45)$ & $32.55(5.04)$ & $32.02(5.41)$ & .972 & $32.72(3.92)$ & $33.75(4.57)$ & $33.45(4.91)$ & .052 \\
\hline $6 \mathrm{M}$ & $35.05(6.72)$ & $34.32(5.50)$ & $36.36(6.90)$ & & $36.37(4.77)$ & $35.59(3.42)$ & $39.27(4.87)$ & \\
\hline $9 \mathrm{M}$ & $35.62(6.81)$ & $35.07(5.89)$ & $34.49(5.95)$ & & $38.24(5.48)$ & $36.25(5.14)$ & $36.31(4.17)$ & \\
\hline $12 \mathrm{M}$ & $34.11(6.29)$ & $34.61(6.05)$ & 34.89 (6.45) & & $35.41(4.84)$ & $35.82(5.67)$ & 37.04 (5.28) & \\
\hline
\end{tabular}

${ }^{\mathrm{a} P}$-values for significance of interaction effects between exercise group and time via the generalized estimating equation.

FMD = flow mediated dilation; PWV = pulse wave velocity; $\mathrm{HDL}-\mathrm{C}=$ high-density lipoprotein cholesterol; IMT = intima media thickness; LDL-C=low-density lipoprotein cholesterol; $\mathrm{SD}=$ standard deviation; $\mathrm{TC}=$ total cholesterol; $\mathrm{VO}_{2 \max }=$ maximal oxygen consumption; $\mathrm{WC}=$ waist circumference.

${ }^{*} \mathrm{P}<.05$ is for a significant difference in fasting glucose levels by exercise mode at 12 months.

found significant time effects for body weight $(\mathrm{p}<.001)$, lean body mass $(\mathrm{p}=.040)$, waist circumference $(\mathrm{p}<.001)$, total cholesterol $(\mathrm{p}=.006)$, LDL cholesterol $(\mathrm{p}=.020)$, and HDL cholesterol levels $(\mathrm{p}<.001)$, fasting glucose levels $(\mathrm{p}<.001)$, and $\mathrm{VO}_{2 \max }(\mathrm{p}<.001)$, based on the modified ITT analysis results. In other words, regardless of the exercise modes, PWV, IMT, body weight, waist circumference, and total and LDL cholesterol levels significantly decreased over time in the diet-plus-exercise intervention; $\mathrm{HDL}$ cholesterol level and $\mathrm{VO}_{2 \max }$ significantly increased over time in the diet-plus-exercise intervention. Specifically, changes in body weight, waist circumference, HDL cholesterol level, and $\mathrm{VO}_{2 \max }$ primarily showed linear trends. The IMT and fasting glucose levels showed quadratic curve trends, while PWV and total and LDL cholesterol levels showed cubic curve trends, based on the modified ITT analysis results. The findings from the PP analyses almost closely matched those from the modified ITT analyses.

\section{Discussion}

The exercise mode did not have significant differential effects on FMD, PWV, and IMT in the diet-plus-exercise intervention of a 12-month weight management program. However, the exercise mode had a significant differential effect on fasting glucose levels; combination exercise lowered fasting glucose levels more effectively than aerobic exercise. Meanwhile, there were significant time effects of the diet-plus-exercise intervention, i.e., reductions in PWV and IMT in cubic and quadratic trends, respectively, and improvements in body weight; waist circumference; levels of total, LDL, and HDL cholesterol; and cardiorespiratory fitness in linear, quadratic, or cubic trends. 
Table 3 Time effects on markers of subclinical atherosclerosis and anthropometric and cardiometabolic profiles

\begin{tabular}{|c|c|c|c|c|}
\hline & \multicolumn{2}{|c|}{ Modified intention-to-teat analysis $(\mathrm{N}=92)$} & \multicolumn{2}{|c|}{ Per-protocol analysis $(\mathrm{N}=49)$} \\
\hline & Mean (SD) & $P^{a}$ & Mean (SD) & $P^{a}$ \\
\hline \multicolumn{5}{|c|}{ FMD $(\mathrm{mm})$} \\
\hline $3 \mathrm{M}$ & $10.93(3.57)$ & .676 & $11.23(3.67)$ & .628 \\
\hline $6 \mathrm{M}$ & $11.33(3.84)$ & & $1171(4.17)$ & \\
\hline $9 \mathrm{M}$ & $11.41(4.20)$ & & $11.54(4.57)$ & \\
\hline $12 \mathrm{M}$ & $11.11(4.18)$ & & $10.93(4.47)$ & \\
\hline \multicolumn{5}{|c|}{ PWV (m/sec) } \\
\hline $3 \mathrm{M}$ & $7.89(1.14)$ & $.048^{\ddagger}$ & $7.60(0.87)$ & .055 \\
\hline $6 \mathrm{M}$ & $7.72(1.19)$ & & $7.36(0.88)$ & \\
\hline $9 \mathrm{M}$ & $7.88(1.19)$ & & $7.57(1.00)$ & \\
\hline $12 \mathrm{M}$ & $7.87(1.15)$ & & $7.56(0.88)$ & \\
\hline \multicolumn{5}{|c|}{ IMT (mm) } \\
\hline $3 \mathrm{M}$ & $0.70(0.16)$ & $.018^{\dagger \neq}$ & $0.71(0.19)$ & .042 \\
\hline $6 \mathrm{M}$ & $0.67(0.14)$ & & $0.67(0.16)$ & \\
\hline $9 \mathrm{M}$ & $0.68(0.13)$ & & $0.70(0.13)$ & \\
\hline $12 \mathrm{M}$ & $0.69(0.13)$ & & $0.71(0.13)$ & \\
\hline \multicolumn{5}{|c|}{ Body weight (kg) } \\
\hline $3 \mathrm{M}$ & $70.45(10.86)$ & $<.001^{*+\ddagger}$ & $67.84(9.46)$ & $<.001^{*+\neq}$ \\
\hline $6 \mathrm{M}$ & $68.95(11.37)$ & & $65.81(9.87)$ & \\
\hline $9 \mathrm{M}$ & $68.96(11.41)$ & & $65.08(7.84)$ & \\
\hline $12 \mathrm{M}$ & $69.04(11.55)$ & & $66.03(10.14)$ & \\
\hline \multicolumn{5}{|c|}{ Lean body mass (kg) } \\
\hline $3 \mathrm{M}$ & $45.29(4.97)$ & $.040^{*}$ & $44.65(5.01)$ & .153 \\
\hline $6 \mathrm{M}$ & $45.14(5.02)$ & & $44.40(5.08)$ & \\
\hline $9 \mathrm{M}$ & $45.08(4.96)$ & & $44.10(4.29)$ & \\
\hline $12 \mathrm{M}$ & $44.99(4.89)$ & & $44.28(4.86)$ & \\
\hline \multicolumn{5}{|l|}{ WC (cm) } \\
\hline $3 \mathrm{M}$ & 92.80 ( 9.21$)$ & $<.001^{*}+\neq$ & $89.88(7.01)$ & $<.001 \dagger$ \\
\hline $6 \mathrm{M}$ & $90.82(10.10)$ & & $87.09(7.95)$ & \\
\hline $9 \mathrm{M}$ & 90.85 (10.08) & & $87.14(7.26)$ & \\
\hline $12 \mathrm{M}$ & 91.39 ( 9.81) & & $88.42(7.60)$ & \\
\hline \multicolumn{5}{|c|}{ Systolic BP(mmHg) } \\
\hline $3 \mathrm{M}$ & $114.30(15.41)$ & .245 & $111.57(12.21)$ & .253 \\
\hline $6 \mathrm{M}$ & $112.77(14.80)$ & & $110.03(13.00)$ & \\
\hline $9 \mathrm{M}$ & $113.59(14.18)$ & & $110.43(10.65)$ & \\
\hline $12 \mathrm{M}$ & 113.08 (14.69) & & $109.57(11.51)$ & \\
\hline \multicolumn{5}{|c|}{$\mathrm{TC}(\mathrm{mg} / \mathrm{dL})$} \\
\hline $3 \mathrm{M}$ & $196.93(38.15)$ & $.006^{\ddagger}$ & $195.40(42.20)$ & $.013^{\ddagger}$ \\
\hline $6 \mathrm{M}$ & $196.73(36.47)$ & & $192.33(37.43)$ & \\
\hline $9 \mathrm{M}$ & $203.46(38.37)$ & & $201.86(41.14)$ & \\
\hline $12 \mathrm{M}$ & 199.39 (41.15) & & 194.66 (45.70) & \\
\hline
\end{tabular}




\begin{tabular}{|c|c|c|c|c|}
\hline \multicolumn{5}{|c|}{ LDL-C (mg/dL) } \\
\hline $3 \mathrm{M}$ & $118.64(35.38)$ & \multirow[t]{4}{*}{$.020^{\ddagger}$} & $118.13(41.36)$ & \multirow[t]{4}{*}{$.034^{\ddagger}$} \\
\hline $6 \mathrm{M}$ & $118.52(31.52)$ & & $115.88(34.15)$ & \\
\hline $9 \mathrm{M}$ & $123.07(36.68)$ & & $121.74(41.40)$ & \\
\hline $12 \mathrm{M}$ & $118.76(38.17)$ & & $115.65(43.25)$ & \\
\hline \multicolumn{5}{|c|}{$\mathrm{HDL}-\mathrm{C}(\mathrm{mg} / \mathrm{dL})$} \\
\hline $3 \mathrm{M}$ & $52.07(11.78)$ & \multirow[t]{4}{*}{$<.001^{*}$} & $51.59(12.63)$ & \multirow[t]{4}{*}{$<.001^{*}$} \\
\hline $6 \mathrm{M}$ & $54.01(12.54)$ & & $53.97(14.28)$ & \\
\hline $9 \mathrm{M}$ & $55.34(12.25)$ & & $56.92(13.64)$ & \\
\hline $12 \mathrm{M}$ & $56.47(13.26)$ & & $57.59(14.06)$ & \\
\hline \multicolumn{5}{|c|}{ Triglycerides (mg/dL) } \\
\hline $3 \mathrm{M}$ & $131.12(77.41)$ & \multirow[t]{4}{*}{.289} & $128.39(90.15)$ & \multirow[t]{4}{*}{.197} \\
\hline $6 \mathrm{M}$ & $121.00(62.72)$ & & $112.40(60.51)$ & \\
\hline $9 \mathrm{M}$ & $125.25(71.84)$ & & $115.99(77.68)$ & \\
\hline $12 \mathrm{M}$ & $120.55(68.62)$ & & $106.64(68.35)$ & \\
\hline \multicolumn{5}{|c|}{ Fasting glucose (mg/dL) } \\
\hline $3 \mathrm{M}$ & $90.65(13.13)$ & \multirow[t]{4}{*}{$<.001^{\dagger}$} & 89.09 (9.52) & \multirow[t]{4}{*}{$.034^{*+}$} \\
\hline $6 \mathrm{M}$ & $87.42(12.54)$ & & $85.13(9.33)$ & \\
\hline $9 \mathrm{M}$ & $87.29(14.41)$ & & $85.60(13.13)$ & \\
\hline $12 \mathrm{M}$ & $89.62(13.70)$ & & $88.23(11.93)$ & \\
\hline \multicolumn{5}{|c|}{$\mathrm{VO}_{2 \max }(\mathrm{mL} / \mathrm{kg} / \min )$} \\
\hline $3 \mathrm{M}$ & $32.06(5.27)$ & \multirow[t]{4}{*}{$<.001^{*+\neq}$} & $33.23(4.34)$ & \multirow[t]{4}{*}{$<.001^{*+}$} \\
\hline $6 \mathrm{M}$ & $35.24(6.44)$ & & $37.04(4.63)$ & \\
\hline $9 \mathrm{M}$ & $35.14(6.26)$ & & $37.05(4.97)$ & \\
\hline $12 \mathrm{M}$ & $34.48(6.21)$ & & $36.02(5.15)$ & \\
\hline
\end{tabular}

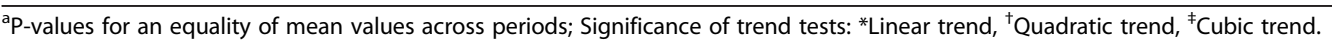
FMD = flow mediated dilation; PWV = pulse wave velocity; IMT = intima media thickness; HDL-C = high-density lipoprotein cholesterol; LDL-C = low-density lipoprotein cholesterol; $\mathrm{SD}=$ standard deviation; $\mathrm{TC}=$ total cholesterol; $\mathrm{VO}_{2 \max }=$ maximal oxygen consumption; $\mathrm{WC}=$ waist circumference.

We found neither differential effects of exercise modes nor time effects of the diet-plus-exercise intervention on FMD in women with abdominal obesity. Several studies have previously reported the beneficial effects of both aerobic and resistance exercise on FMD. Aerobic exercise training is well known to improve FMD [30-32], although only a few studies have reported the effects of aerobic exercise on FMD in obese individuals. Watts et al. [33] reported that FMD was significantly greater among trained obese children compared with untrained obese children after 8 weeks of aerobic exercise training [33]. Similarly, either resistance or combination exercise training may be effective in improving endothelial function, although this was found in small-scale studies, thereby necessitating further confirmation [13,34,35]. In a study of 30 overweight women, Olson et al. [13] reported that FMD improved significantly in a resistance exercise group compared with a control group after a 1-year resistance training intervention [13]. Maiorana et al. [35] reported in a crossover study that a combination of aerobic and resistance exercise for 8 weeks significantly increased FMD among 16 patients with type 2 diabetes mellitus [35]. Nevertheless, only a few studies have reported the differential effects of exercise mode [36]. Kwon et al. [36] found that aerobic exercise provide greater improvement in FMD than resistance exercise among 40 overweight women with type 2 diabetes mellitus in a comparison study of exercise mode [36].

In the present study, the non-significant differential effects of exercise mode on FMD for women with abdominal obesity may be explained by the complex effects of both exercise and diet in our study design. In previous studies, the effects of exercise training combined with dietary interventions have thus far yielded mixed results among obese individuals. Hamdy et al. [37] reported an improvement in the FMD in 24 obese individuals after a 6-month lifestyle modification consisting of both energy-restricted diet and aerobic exercise training [37]. In contrast, Wycherley et al. [19] did not find any significant improvement in the FMD in 29 obese patients with 
type II diabetes after a 12-week caloric restriction with aerobic exercise training [19]. Such conflicting findings might be attributable to distinct characteristics of FMD from other measures of subclinical atherosclerosis such as carotid IMT. The Firefighters and their Endothelium study (2005) [38] showed that the FMD had no significant correlation with carotid IMT among middle-aged men who were at low-to-moderate risk of cardiovascular diseases [38]. FMD is a well-standardized measure of endothelial function that is considered an early manifestation of atherosclerosis [39]. Furthermore, FMD is an acute indicator of vascular function, as opposed to carotid IMT, which is a chronic indicator of vascular structural abnormalities. Therefore, changes in FMD may, in part, result from acute influences such as withinindividual variations in the exposure to the complex effects of either exercise or diet (i.e., changes in body weight or cardiometabolic risk factors) when they were measured. Meanwhile, non-significant changes in the FMD found in the present study may also be explained by the normal range $(\geq 5.5 \%)$ of the FMD values at baseline (10.8\%; range, $4.0-18.0 \%$ in our data). Swift et al. [31] reported a significant improvement in the FMD in obese women with impaired FMD, but not in those with normal FMD at baseline after a 6-month aerobic exercise training program [31].

We found no differential effect of exercise mode on aortic PWV, but a significant reduction in aortic PWV with the diet-plus-exercise intervention over time. Aerobic exercise training has previously been reported to decrease aortic PWV in a few studies, although the findings were limited to healthy men. Hayashi et al. [40] reported that 16 weeks of aerobic training with moderate-intensity walking and jogging significantly decreased aortic PWV in 17 sedentary middle-aged men [40]. Collier et al. [41] found that aerobic exercise led to a decrease in aortic PWV, whereas resistance exercise led to a significant increase in aortic PWV after 4-week training among 30 middle-aged individuals with prehypertension or stage1 hypertension [41]. However, such increases in aortic PWV with resistance training need to be further clarified. In fact, we did not find any increase in aortic PWV in the resistance exercise group. Similarly, a meta-analysis by Miyachi showed that resistance training may not be associated with increases in aortic PWV in middle-aged subjects [42]. Meanwhile, our data showed that the reduction in aortic PWV peaked at 6 months in the entire intervention and rebounded after the next 6 months in the diet-plus-exercise intervention in a cubic curve trend; this trend appeared to parallel with the weight loss trend in our data. Dengo et al. [43] reported that a reduction in aortic PWV correlated with reductions in total body and abdominal adiposity among overweight and obese adults [43]. In this context, the reduction of aortic PWV may be primarily related to exercise-induced energy expenditure contributing to weight loss rather than exercise mode.

We did not find any differential effect of exercise modes on carotid IMT. Instead, we found a significant regression in carotid IMT with the diet-plus-exercise intervention as a time effect. Similarly, Spence et al. [44] reported that there was no significant differential effect of aerobic versus resistance exercise training on carotid IMT, but there was a significant time effect among 23 young healthy male subjects with 24 weeks of exercise training [44]. Dutheil et al. [17] investigated the effects of combination exercise on carotid IMT according to exercise intensity, and reported that moderate-resistance high-aerobic exercise was more effective for the regression of carotid IMT than moderate-resistance moderate-aerobic exercise [17]. Thus, we speculate that the effects of exercise training on carotid IMT may be influenced by exercise intensity but not by exercise mode.

In our findings, the regression effect of exercise training on carotid IMT (i.e., a time effect) was not linear over time, with a peak at 6 months that was not maintained to 12 months, consistent with the findings of a previous study [45]. There was little evidence for beneficial effects of a lifestyle intervention (i.e., either diet-alone or diet-plusexercise intervention) on carotid IMT among overweight and obese individuals [45-47]. Fuentes et al. [45] reported a significant regression in carotid IMT over a 24-month follow-up with a diet-induced weight-loss intervention involving 60 obese individuals [45]. Carotid IMT is a vascular measure of structural changes in the carotid arteries, so a reduction in carotid IMT may require longduration interventions. Thus, the underlying mechanism of these short-term changes in carotid IMT with the diet-plus-exercise intervention need to be further clarified, but we could speculate that the changes are the result of various favorable changes in the cardiometabolic risk factors that occur concomitantly with weight loss induced by the diet-plus-exercise intervention.

Finally, we found a significant differential effect of exercise mode on fasting glucose levels but not on vascular measures (i.e., FMD, PWV, and IMT); specifically, combination of aerobic and resistance exercises was significantly more effective in lowering fasting glucose levels than aerobic exercise alone in the diet-plus-exercise intervention. The beneficial effect of a combination of aerobic and resistance exercises on glucose control has been previously reported only in patients with type 2 diabetes $[21,48,49]$ but not in overweight and obese individuals. Snowling et al. [49] concluded, from their meta-analysis, that aerobic, resistance, and combination exercises had small-to-moderate beneficial effects on glucose control in patients with type 2 diabetes; particularly, compared to aerobic exercise, combination exercise was 
found to lower fasting glucose levels, albeit to a small extent [49]. Meanwhile, the conflicting effects of exercise mode on fasting glucose levels versus vascular measures may be explained by different exercise-induced physiological responses. The beneficial effect of combination exercise on fasting glucose levels may be attributable to an additional beneficial effect of resistance exercise to aerobic exercise. Physiologically, similar to aerobic exercise, resistance exercise can increase glucose uptake and glycogen repletion in skeletal muscle following exercise by promoting contraction-mediated GLUT4 translocation with increased activation of AMP-activated protein kinase [50]. In this context, both aerobic and resistance exercise may provide a synergistic effect on glucose metabolism. In contrast, non-significant differential effects of exercise mode on vascular measures (i.e., PWV and IMT) may indicate that changes in vascular measures influenced by vascular tone and remodeling with exercise training are not influenced by exercise mode but instead by other components such as exercise intensity or exercise expenditure [17].

This study, to the best of our knowledge, is the first to report on the effects of exercise modes in a weight management intervention on major markers of subclinical atherosclerosis such as endothelial dysfunction, arterial stiffness, and carotid IMT. Nevertheless, this study has several limitations. First, the attrition rate in the study was $55 \%$ over 12 months, and this may have led to a bias influencing the validity of results. However, the present study was initiated and implemented as a communitybased program and its attrition rate was similar to that in previous studies conducted as community-based programs [51]. Second, of the participants included in the modified ITT analysis ( $\mathrm{n}=92), 68 \%$ had a menstrual cycle. FMD may modulate in response to changing hormonal patterns during the menstrual cycle [52]. Lack of consideration of the effects of the menstrual cycle in vascular measurements might have underestimated or overestimated changes in FMD. Third, since all the participants in present study were Korean women, the results cannot be generalized to men or other ethnic groups.

\section{Conclusions}

For women with abdominal obesity, a combination of aerobic and resistance exercise may be preferable to a single mode (i.e., aerobic or resistance exercise) for effective glucose control. Regardless of exercise mode, exercise interventions combined with dietary interventions may be beneficial for reducing the risk of subclinical atherosclerosis and cardiometabolic risk in women with abdominal obesity. Such beneficial effects of exercise training may not be differentiated by exercise mode but by other exercise components such as exercise intensity or expenditure.

\section{Competing interests}

The authors declare that they have no competing interests.

\section{Authors' contributions}

The authors' contributions were as follows: JC developed the hypothesis of this study and prepared the manuscript draft; JC, JHC, and SYJ were involved in data collection; JC and JL performed the data analyses; SYJ, AS and LEB provided expert consultation on data interpretation and helped to draft the manuscript. All authors were involved in the review and revision of the manuscript and gave approval for the final version to be published.

\section{Acknowledgements}

This research was supported by the Basic Science Research Program through the National Research Foundation of Korea (NRF) funded by the Ministry of Education, Science and Technology (No. 2010-0022022).

\section{Author details}

${ }^{1}$ College of Nursing, Korea University, Seoul, South Korea. ${ }^{2}$ Department of Biostatistics, College of Medicine, Korea University, Seoul, South Korea. ${ }^{3}$ School of Nursing and Epidemiology and Clinical and Translational Science Institute, University of Pittsburgh, Pennsylvania, USA. ${ }^{4}$ Department of Epidemiology, Graduate School of Public Health, University of Pittsburgh, Pennsylvania, USA. ${ }^{5}$ College of Arts and Physical Education, University of Seoul, Seoul, South Korea.

Received: 26 March 2014 Accepted: 1 July 2014

Published: 10 July 2014

\section{References}

1. Flint AJ, Rexrode KM, Hu FB, Glynn RJ, Caspard H, Manson JE, Willett WC, Rimm EB: Body mass index, waist circumference, and risk of coronary heart disease: a prospective study among men and women. Obes Res Clin Pract 2010, 4(3):e171-e181.

2. Arcaro G, Zamboni M, Rossi L, Turcato E, Covi G, Armellini F, Bosello O, Lechi A: Body fat distribution predicts the degree of endothelial dysfunction in uncomplicated obesity. Int J Obes Relat Metab Disord 1999, 23(9):936-942.

3. Recio-Rodriguez II, Gomez-Marcos MA, Patino-Alonso MC, Agudo-Conde C, Rodriguez-Sanchez E, Garcia-Ortiz L: Abdominal obesity vs general obesity for identifying arterial stiffness, subclinical atherosclerosis and wave reflection in healthy, diabetics and hypertensive. BMC Cardiovasc Disord 2012, 12:3.

4. Asicioglu E, Kahveci A, Arikan H, Koc M, Tuglular S, Ozener Cl: Waist circumference is associated with carotid intima media thickness in peritoneal dialysis patients. Int Urol Nephrol 2013, 45(5):1437-1443.

5. Rexrode KM, Carey VJ, Hennekens CH, Walters EE, Colditz GA, Stampfer MJ, Willett WC, Manson JE: Abdominal adiposity and coronary heart disease in women. JAMA 1998, 280(21):1843-1848.

6. Rexrode KM, Buring JE, Manson JE: Abdominal and total adiposity and risk of coronary heart disease in men. Int J Obes Relat Metab Disord 2001, 25(7):1047-1056.

7. Arsenault BJ, Rana JS, Lemieux I, Despres JP, Kastelein JJ, Boekholdt SM, Wareham NJ, Khaw KT: Physical inactivity, abdominal obesity and risk of coronary heart disease in apparently healthy men and women. Int J Obes (Lond) 2010, 34(2):340-347.

8. Wu T, Gao X, Chen M, van Dam RM: Long-term effectiveness of diet-plusexercise interventions vs. diet-only interventions for weight loss: a meta-analysis. Obes Rev 2009, 10(3):313-323.

9. Kelley GA, Kelley KS: Effects of diet, aerobic exercise, or both on non-HDL$C$ in adults: a meta-analysis of randomized controlled trials. Cholesterol 2012, 2012:840935.

10. Wing RR, Venditti $E$, Jakicic JM, Polley BA, Lang W: Lifestyle intervention in overweight individuals with a family history of diabetes. Diabetes Care 1998, 21(3):350-359.

11. Hagan RD, Upton SJ, Wong L, Whittam J: The effects of aerobic conditioning and/or caloric restriction in overweight men and women. Med Sci Sports Exerc 1986, 18(1):87-94.

12. Beck DT, Martin JS, Casey DP, Braith RW: Exercise training reduces peripheral arterial stiffness and myocardial oxygen demand in young prehypertensive subjects. Am J Hypertens 2013, 26(9):1093-1102.

13. Olson TP, Dengel DR, Leon AS, Schmitz KH: Moderate resistance training and vascular health in overweight women. Med Sci Sports Exerc 2006, 38(9):1558-1564.

14. Tjonna AE, Stolen TO, Bye A, Volden M, Slordahl SA, Odegard R, Skogvoll E, Wisloff $U$ : Aerobic interval training reduces cardiovascular risk factors 
more than a multitreatment approach in overweight adolescents. Clin Sci (Lond) 2009, 116(4):317-326.

15. Guimaraes GV, Ciolac EG, Carvalho VO, D'Avila VM, Bortolotto LA, Bocchi EA: Effects of continuous vs. interval exercise training on blood pressure and arterial stiffness in treated hypertension. Hypertens Res 2010, 33(6):627-632.

16. Goodpaster BH, Delany JP, Otto AD, Kuller L, Vockley J, South-Paul JE, Thomas SB, Brown J, McTigue K, Hames KC, Lang W, Jakicic JM: Effects of diet and physical activity interventions on weight loss and cardiometabolic risk factors in severely obese adults: a randomized trial. JAMA 2010, 304(16):1795-1802.

17. Dutheil F, Lac G, Lesourd B, Chapier R, Walther G, Vinet A, Sapin V, Verney J, Ouchchane L, Duclos M, Obert P, Courteix D, Obert P, Courteix D: Different modalities of exercise to reduce visceral fat mass and cardiovascular risk in metabolic syndrome: the RESOLVE randomized trial. Int J Cardio/ 2013, 168(4):3634-3642.

18. McKelvie RS, McCartney N, Tomlinson C, Bauer R, MacDougall JD: Comparison of hemodynamic responses to cycling and resistance exercise in congestive heart failure secondary to ischemic cardiomyopathy. Am J Cardiol 1995, 76(12):977-979.

19. Wycherley TP, Brinkworth GD, Noakes M, Buckley JD, Clifton PM: Effect of caloric restriction with and without exercise training on oxidative stress and endothelial function in obese subjects with type 2 diabetes. Diabetes Obes Metab 2008, 10(11):1062-1073.

20. Schwingshackl L, Dias S, Strasser B, Hoffmann G: Impact of different training modalities on anthropometric and metabolic characteristics in overweight/obese subjects: a systematic review and network metaanalysis. PLoS One 2013, 8(12):e82853.

21. Sigal RJ, Kenny GP, Boule NG, Wells GA, Prud'homme D, Fortier M, Reid RD, Tulloch $\mathrm{H}$, Coyle D, Phillips $\mathrm{P}$, Jennings $A$, Jaffey J: Effects of aerobic training, resistance training, or both on glycemic control in type 2 diabetes: a randomized trial. Ann Intern Med 2007, 147(6):357-369.

22. Foster-Schubert KE, Alfano CM, Duggan CR, Xiao L, Campbell KL, Kong A, Bain CE, Wang CY, Blackburn GL, MCTiernan A: Effect of diet and exercise, alone or combined, on weight and body composition in overweight-to-obese postmenopausal women. Obesity (Silver Spring) 2012, 20(8):1628-1638.

23. Lee SY, Park HS, Kim DJ, Han JH, Kim SM, Cho GJ, Kim DY, Kwon HS, Kim SR, Lee CB, Oh SJ, Park CY, Yoo HJ: Appropriate waist circumference cutoff points for central obesity in Korean adults. Diabetes Res Clin Pract 2007, 75(1):72-80.

24. Cho JH, Jae SY, Choo IL, Choo J: Health-promoting behaviour among women with abdominal obesity: a conceptual link to social support and perceived stress. J Adv Nurs 2014, 70(6):1381-1390.

25. Burke LE, Wang J: Treatment strategies for overweight and obesity. J Nurs Scholarsh 2011, 43(4):368-375.

26. Friedewald WT, Levy RI, Fredrickson DS: Estimation of the concentration of low-density lipoprotein cholesterol in plasma, without use of the preparative ultracentrifuge. Clin Chem 1972, 18(6):499-502

27. Kline GM, Porcari JP, Hintermeister R, Freedson PS, Ward A, McCarron RF, Ross J, Rippe JM: Estimation of VO2max from a one-mile track walk, gender, age, and body weight. Med Sci Sports Exerc 1987, 19(3):253-259.

28. Third Report of the Expert Panel on Detection, Evaluation, and Treatment of the High Blood Cholesterol in Adults (Adult Treatment Panel III): Executive Summary. http://www.nhlbi.nih.gov/guidelines/cholesterol/atp_iii.htm.

29. Grundy SM, Cleeman Jl, Daniels SR, Donato KA, Eckel RH, Franklin BA, Gordon DJ, Krauss RM, Savage PJ, Smith SC Jr, Spertus JA, Costa F: Diagnosis and management of the metabolic syndrome: an American Heart Association/National Heart, Lung, and Blood Institute Scientific Statement. Circulation 2005, 112(17):2735-2752.

30. McDermott MM, Ades P, Guralnik JM, Dyer A, Ferrucci L, Liu K, Nelson M, Lloyd-Jones D, Van Horn L, Garside D, Kibbe M, Domanchuk K, Stein JH, Liao Y, Tao H, Green D, Pearce WH, Schneider JR, McPherson D, Laing ST, McCarthy WJ, Shroff A, Criqui MH: Treadmill exercise and resistance training in patients with peripheral arterial disease with and without intermittent claudication: a randomized controlled trial. JAMA 2009, 301(2):165-174.

31. Swift DL, Earnest CP, Blair SN, Church TS: The effect of different doses of aerobic exercise training on endothelial function in postmenopausal women with elevated blood pressure: results from the DREW study. $\mathrm{Br} J$ Sports Med 2012, 46(10):753-758.

32. DeSouza CA, Shapiro LF, Clevenger CM, Dinenno FA, Monahan KD, Tanaka $H$, Seals DR: Regular aerobic exercise prevents and restores age-related declines in endothelium-dependent vasodilation in healthy men. Circulation 2000, 102(12):1351-1357.
33. Watts K, Beye P, Siafarikas A, O'Driscoll G, Jones TW, Davis EA, Green DJ: Effects of exercise training on vascular function in obese children. J Pediatr 2004, 144(5):620-625.

34. Okada S, Hiuge A, Makino H, Nagumo A, Takaki H, Konishi H, Goto Y, Yoshimasa Y, Miyamoto Y: Effect of exercise intervention on endothelial function and incidence of cardiovascular disease in patients with type 2 diabetes. J Atheroscler Thromb 2010, 17(8):828-833.

35. Maiorana A, O'Driscoll G, Cheetham C, Dembo L, Stanton K, Goodman C, Taylor $\mathrm{R}$, Green D: The effect of combined aerobic and resistance exercise training on vascular function in type 2 diabetes. J Am Coll Cardiol 2001, 38(3):860-866.

36. Kwon HR, Min KW, Ahn HJ, Seok HG, Lee JH, Park GS, Han KA: Effects of aerobic exercise vs. resistance training on endothelial function in women with type 2 diabetes mellitus. Diabetes Metab J 2011, 35(4):364-373.

37. Hamdy O, Ledbury S, Mullooly C, Jarema C, Porter S, Ovalle K, Moussa A, Caselli A, Caballero AE, Economides PA, Veves A, Horton ES: Lifestyle modification improves endothelial function in obese subjects with the insulin resistance syndrome. Diabetes Care 2003, 26(7):2119-2125.

38. Yan RT, Anderson TJ, Charbonneau F, Title L, Verma S, Lonn E: Relationship between carotid artery intima-media thickness and brachial artery flowmediated dilation in middle-aged healthy men. J Am Coll Cardiol 2005, 45(12):1980-1986.

39. Landmesser $U$, Hornig B, Drexler $\mathrm{H}$ : Endothelial function: a critical determinant in atherosclerosis? Circulation 2004, 109(21 Suppl 1):|127-||133.

40. Hayashi K, Sugawara J, Komine H, Maeda S, Yokoi T: Effects of aerobic exercise training on the stiffness of central and peripheral arteries in middle-aged sedentary men. Jpn J Physiol 2005, 55(4):235-239.

41. Collier SR, Kanaley JA, Carhart R Jr, Frechette V, Tobin MM, Hall AK, Luckenbaugh AN, Fernhall B: Effect of 4 weeks of aerobic or resistance exercise training on arterial stiffness, blood flow and blood pressure in pre- and stage-1 hypertensives. J Hum Hypertens 2008, 22(10):678-686.

42. Miyachi M: Effects of resistance training on arterial stiffness: a metaanalysis. Br J Sports Med 2013, 47(6):393-396.

43. Dengo AL, Dennis EA, Orr JS, Marinik EL, Ehrlich E, Davy BM, Davy KP: Arterial destiffening with weight loss in overweight and obese middleaged and older adults. Hypertension 2010, 55(4):855-861

44. Spence AL, Carter HH, Naylor LH, Green DJ: A prospective randomized longitudinal study involving 6 months of endurance or resistance exercise: conduit artery adaptation in humans. J Physiol 2013, 591(Pt 5):1265-1275.

45. de las Fuentes L, Waggoner AD, Mohammed BS, Stein Rl, Miller BV 3rd, Foster GD, Wyatt HR, Klein S, Davila-Roman VG: Effect of moderate diet-induced weight loss and weight regain on cardiovascular structure and function. J Am Coll Cardiol 2009, 54(25)):2376-2381.

46. Mavri A, Stegnar M, Sentocnik JT, Videcnik V: Impact of weight reduction on early carotid atherosclerosis in obese premenopausal women. Obes Res 2001, 9(9):511-516

47. Thijssen DH, Cable NT, Green DJ: Impact of exercise training on arterial wall thickness in humans. Clin Sci (Lond) 2012, 122(7):311-322.

48. Church TS, Blair SN, Cocreham S, Johannsen N, Johnson W, Kramer K, Mikus CR, Myers V, Nauta M, Rodarte RQ, Sparks L, Thompson A, Earnest CP: Effects of aerobic and resistance training on hemoglobin A1c levels in patients with type 2 diabetes: a randomized controlled trial. JAMA 2010, 304(20):2253-2262.

49. Snowling NJ, Hopkins WG: Effects of different modes of exercise training on glucose control and risk factors for complications in type 2 diabetic patients: a meta-analysis. Diabetes Care 2006, 29(11):2518-2527.

50. Dreyer HC, Drummond MJ, Glynn EL, Fujita S, Chinkes DL, Volpi E, Rasmussen BB: Resistance exercise increases human skeletal muscle AS160/TBC1D4 phosphorylation in association with enhanced leg glucose uptake during postexercise recovery. J App/ Physiol 2008, 105(6):1967-1974.

51. Graffagnino CL, Falko JM, La Londe M, Schaumburg J, Hyek MF, Shaffer LE, Snow R, Caulin-Glaser T: Effect of a community-based weight management program on weight loss and cardiovascular disease risk factors. Obesity (Silver Spring) 2006, 14(2):280-288.

52. Williams MR, Westerman RA, Kingwell BA, Paige J, Blombery PA, Sudhir K, Komesaroff PA: Variations in endothelial function and arterial compliance during the menstrual cycle. J Clin Endocrinol Metab 2001, 86(11):5389-5395.

\section{doi:10.1186/1471-2261-14-82}

Cite this article as: Choo et al:: Effects of weight management by exercise modes on markers of subclinical atherosclerosis and cardiometabolic profile among women with abdominal obesity: a randomized controlled trial. BMC Cardiovascular Disorders 2014 14:82. 